\title{
Odontogenic epithelial stem cells: hidden sources
}

\author{
Sivan Padma Priya ${ }^{1,2}$, Akon Higuchi ${ }^{3,4,5}$, Salem Abu Fanas ${ }^{6}$, Mok Pooi Ling ${ }^{7}$, Vasantha Kumari Neela ${ }^{8}$, PM Sunil ${ }^{9,10}$, \\ TR Saraswathi ${ }^{11}$, Kadarkarai Murugan ${ }^{12}$, Abdullah A Alarfaj ${ }^{4}$, Murugan A Munusamy ${ }^{4}$ and Suresh Kumar ${ }^{8,13}$
}

The ultimate goal of dental stem cell research is to construct a bioengineered tooth. Tooth formation occurs based on the well-organized reciprocal interaction of epithelial and mesenchymal cells. The dental mesenchymal stem cells are the best explored, but because the human odontogenic epithelium is lost after the completion of enamel formation, studies on these cells are scarce. The successful creation of a bioengineered tooth is achievable only when the odontogenic epithelium is reconstructed to produce a replica of natural enamel. This article discusses the untapped sources of odontogenic epithelial stem cells in humans, such as those present in the active dental lamina in postnatal life, in remnants of dental lamina (the gubernaculum cord), in the epithelial cell rests of Malassez, and in reduced enamel epithelium. The possible uses of these stem cells in regenerative medicine, not just for enamel formation, are discussed. Laboratory Investigation (2015) 95, 1344-1352; doi:10.1038/labinvest.2015.108; published online 14 September 2015

New treatments for many developmental and degenerative disorders are currently emerging from regenerative medicine, specifically stem cell therapy, which has proven successful in many diseases by providing replacement cells without genetic modification. It is believed that stem cells can be directed to reach a destination if injected or loaded locally to facilitate repair or regeneration. ${ }^{1}$ Dental caries and periodontal problems are two of the most common health problems, afflicting not only the affected individuals but also the economies of developed countries. The dental structures are functionally restored with biocompatible materials, without tissue regeneration. The complex nature of tooth formation (odontogenesis), based on the organized reciprocal interaction of the odontogenic epithelial and cranial neural crest-derived ectomesenchymal tissues, has maintained the functional regeneration of whole teeth, along with their supporting structures, as an unattainable goal in the field of human tooth bioengineering. ${ }^{2}$ There are many more reports about mesenchymal stem cells (MSCs) than about odontogenic epithelial stem cells (OEpSCs). ${ }^{3}$ The OEpSCs are defined as the stem cells involved in tooth development (odontogenesis), which are of outer ectodermal (epithelial) origin and interact reciprocally with the odontogenic MSCs of ectomesenchymal origin. There are sources of OEpSCs that have never been discussed, and their possible retrieval and applications in stem cell research should be revealed. ${ }^{3}$ This article seeks to describe the possibility of using OEpSCs as another source of dental stem cell (DSCs).

\section{Role of OEpSCs during Odontogenesis}

The role of OEpSCs during odontogenesis is indispensable. In 2012, Jussila and Thesleff ${ }^{4}$ discussed the signaling networks and complexity of the regulation involved in odontogenesis including regeneration of human teeth. Human teeth are developed by the well-organized reciprocal interaction of outer ectoderm and cranial neural crest cells (CNCCs) derived from ectomesenchymal cells. The CNCCs are from neural ectoderm (epithelium), which are migrate to the mesenchyme by a process called epithelial-mesenchymal transition (EMT). The CNCCs are more specialized cells because of their ability to form the greater part of the

${ }^{1}$ Department of Basic Medical Science, Ajman University of Science and Technology-Fujairah Campus, Al Fujairah, United Arab Emirates; ${ }^{2}$ Department of Surgical Sciences, Ajman University of Science and Technology, Ajman, United Arab Emirates; ${ }^{3}$ Department of Chemical and Materials Engineering, National Central University, Jhong-li, Taoyuan, Taiwan; ${ }^{4}$ Department of Botany and Microbiology, King Saud University, Riyadh, Saudi Arabia; ${ }^{5}$ Department of Reproduction, National Research Institute for Child Health and Development, Tokyo, Japan; ${ }^{6}$ Department of Surgical Sciences, Ajman University of Science and Technology, Ajman, United Arab Emirates; ${ }^{7}$ Department of Obstetrics and Gynaecology, Faculty of Medicine and Health Sciences, Universiti Putra Malaysia, Selangor, Malaysia; ${ }^{8}$ Department of Medical Microbiology and Parasitology, Universiti Putra Malaysia, Slangor, Malaysia; ${ }^{9}$ Department of Oral Pathology, Sri Anjaneya Institute of Dental Science, Calicut, India; ${ }^{10}$ Director of Stem Cell and Regenerative Medicine Lab, Malabar Medical College, Calicut, India; ${ }^{11}$ Department of Oral Pathology, Tamil Nadu Government Dental College, Chennai, India; ${ }^{12}$ Division of Entomology, Department of Zoology, School of Life Sciences, Bharathiar University, Coimbatore, India and ${ }^{13}$ Genetics and Regenerative Medicine Research Center, Universiti Putra Malaysia, Selangor, Malaysia

Correspondence: Professor SP Priya, MDS, Department of Basic Medical Science and Department of Surgical Sciences, Ajman University of Science and Technology, Ajman, United Arab Emirates or Professor A Higuchi, PhD, Department of Chemical and Materials Engineering, National Central University, No. 300, Jhongda RD., Jhongli 32001, Taoyuan, Taiwan or Professor S Kumar, PhD, Department of Medical Microbiology and Parasitology, Universities Putra Malaysia, Slangor, Malaysia.

E-mail: priyaganu@yahoo.com or higuchi@ncu.edu.tw or sureshkudsc@gmail.com

Received 20 January 2015; revised 22 May 2015; accepted 29 May 2015 
craniofacial structures and the remarkable multilineage differentiation property persisting even in adult tissue.

Figure 1 shows the contents of tooth germ and their relations during embryogenesis, which describes the primary tooth in cap stage and the permanent tooth in bud stage of the odontogenesis. The dental lamina (DL) bestows both the primary and permanent teeth during embryogenesis. These embryonic stage cells with the ability to proceed with the odontogenesis exist even up to the fifth year of postnatal life in the third molar region, but are kept hushed till initiated. The stem cell population at these stages can be explained from the fact that (i) the DL and enamel organ (EO) have OEpSCs, (ii) the dental papilla (DP) has dental pulp stem cells (DPSCs), and (iii) the dental follicle (DF) has DF progenitor cells (Table 1 ).

The DL is a band of ectodermal ingrowth from the outer ectoderm into the mesenchyme in the region of the future dental arch. These odontogenic epithelial ingrowths are the main source of the OEpSC and tooth buds in the respective place for teeth called as EO, the function of which is not only restricted to enamel formation but also to induct the ectomesenchymal cells to form other tissues of the teeth. The EO progresses during different stages of odontogenesis namely bud, cap, and bell, constantly interacting with the ectomesenchymal cells named as DP and DF. EO with DP and DF are collectively named as the tooth germ (Figure 1). The $\mathrm{EO}$ is the inducer for the DP to differentiate the odontoblasts to secrete dentin. The presence of the dentin is the stimulation for the EO to differentiate the enamel-secreting ameloblasts. The absence of EO will make no dentin and the dentin absence will prevent enamel formation.

The cervical region of EO forms Hedwig's epithelial root sheath (HERS) after crown completion. This sheath of OEpSCs is important to induct the root dentin formation as they are doing in the crown. After dentin formation in the root, the HERS dissociates and disintegrates to allow the outer DF cells to contact the dentin to differentiate and secrete the cementum. This process establishes the periodontal attachment between the cementum and the alveolar bone around the developing root. These HERS cells might undergo apoptosis or EMT to rest inside the periodontal ligament as epithelial cells rest of Malassez (ERM). ${ }^{5}$ Animals like mouse having continuously growing anterior teeth possess the HERS throughout the life for unceasing enamel and root formation. However, the active HERS is not available in human after root completion. Humans have two sets of teeth, 20 deciduous and 32 permanent teeth. This same scenario of odontogenesis is repeated for each tooth including the third molar, which is initiated by around 5-6 years of postnatal life. The permanent molars without predecessor teeth are formed by the extension of the DL along with the growth of the jaws.

\section{DENTAL STEM CELLS (DSCS)}

DSCs collectively include the cells of epithelial and mesenchymal origin. Studies related to mesenchymal DSCs of

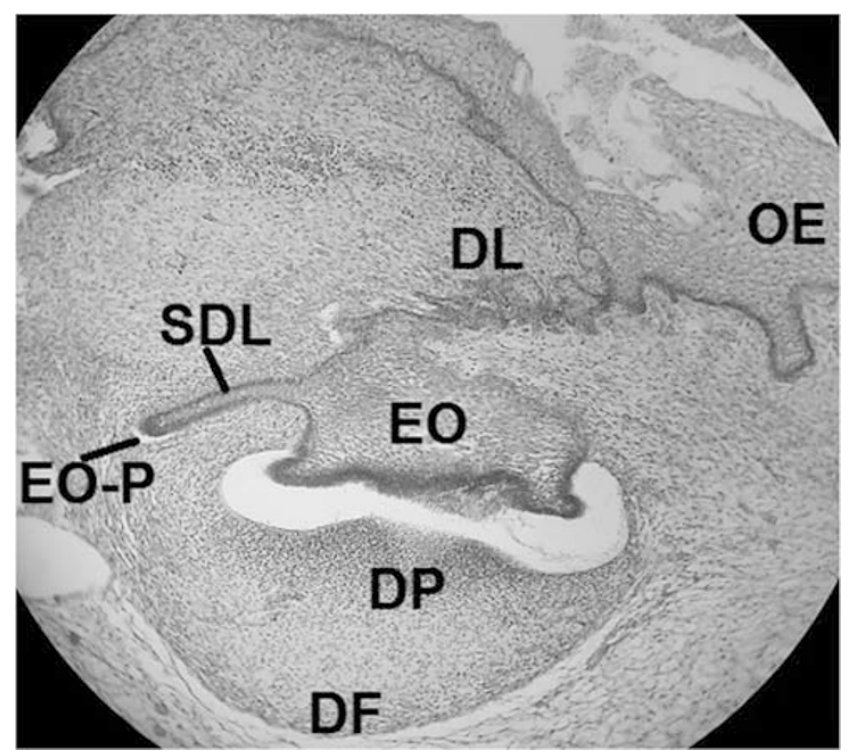

Figure 1 Developing deciduous tooth in cap stage with permanent tooth bud and successional DL in fetus during embryogenesis. The $\mathrm{DL}$, the ingrowth inside the mesenchyme, demonstrates the continuity between the outer epithelium and the EO of the primary (deciduous) in cap stage and permanent (successional) tooth in bud stage. The diverse group of cells in tooth germ is composed of the outer ectoderm-derived $\mathrm{DL}$, which generates the EO and the CNCCs derived DP and DF cells. These primitive cells of the embryonic stage are present up to the fifth year of postnatal life for the formation of the third molar. This explains the presence of the DSC in postnatal life. The stem cell population at these stages can be explained as the DL and EO are having OEPSC, the DP having DPSC and DF having DFPC cells. CNCC, cranial neural crest cell; DF, dental follicle; $\mathrm{DL}$, dental lamina; DP, dental papilla; DSC, dental stem cell; EO, enamel organ; EO-P, enamel organ for permanent teeth; $\mathrm{OE}$, outer ectoderm/ epithelium; SDL, successional dental lamina.

\section{Table 1 Sources and types of dental stem cells}

\begin{tabular}{|c|c|c|c|}
\hline Dental stem cells (DSCs) & Acronym & Type & Source \\
\hline Dental pulp stem cells (DPSCs) & DPSCS & MSCs & Sental pulp \\
\hline $\begin{array}{l}\text { Stem cells from human exfoliated } \\
\text { deciduous teeth (SHED) }\end{array}$ & SHED & MSCs & Sental pulp \\
\hline Stem cells from apical papilla (SCAP) & SCAP & MSCs & $\begin{array}{l}\text { Developing } \\
\text { root end }\end{array}$ \\
\hline $\begin{array}{l}\text { Periodontal ligament stem cells } \\
\text { (PDLSCs) }\end{array}$ & PDLSCS & MSCs & $\begin{array}{l}\text { Periodontal } \\
\text { ligament }\end{array}$ \\
\hline Dental follicle progenitor cells (DFPCs) & DFPCs & MSCS & $\begin{array}{l}\text { Around } \\
\text { developing tooth }\end{array}$ \\
\hline $\begin{array}{l}\text { Gingiva-derived mesenchymal } \\
\text { stem cells (GMSCs) }\end{array}$ & GMSCs & MSCs & Gingiva \\
\hline Epithelial cell rests of Malassez (ERM) & ERM & EpSCs & $\begin{array}{l}\text { Periodontal } \\
\text { ligament }\end{array}$ \\
\hline $\begin{array}{l}\text { Dental pulp pluripotent-like } \\
\text { stem cells (DPPSCs) }\end{array}$ & DPPSCs & MSCs & Dental pulp \\
\hline
\end{tabular}

EpSCs, epithelial stem cells; MSCs, mesenchymal stem cells. 
classification, types, methods of isolation, characterization, advantages, and disadvantages have been explored in several articles. ${ }^{6,7}$ DSCs are particularly attractive for their superior advantages, such as being amenable to ethical collection practices, ${ }^{7}$ easy to access, ${ }^{7}$ and comparatively potent ${ }^{8}$ and having a high proliferation rate and a greater cell number than is common for stem cells, ${ }^{8,9}$ with plenty of sources being available at all stages of life. ${ }^{8}$ Furthermore, many cell lines can be retrieved that are demonstrating good compatibility and attachment to a variety of biomaterials. ${ }^{10,11}$ DSCs are comparatively cost effective. ${ }^{12}$ The DSC sources located so far in teeth are listed in Table 1.3,9,13-17

Many reports have described the multi-potentiality and possible applications of mesenchymal DSCs in dental and non-dental tissue regeneration. ${ }^{18-23}$ DSCs are not just reproducing the pulp and periodontium. Several studies demonstrated their potential to become osteoblast, adipocyte, myoblast (smooth muscles and functional (beating) cardiac cells), endothelial cells, chondroblasts, melanocyte, corneal cells, retinal cells, pancreatic islet cells, neurons, and hepatocytes. ${ }^{18-24}$ Their uniqueness of development from CNCCs explains their multilineage differentiation characteristics.

Clinical trials related to the osteogenic properties of DPSCs that included 3 years of follow-up ${ }^{25}$ and related to periodontal tissue regeneration ${ }^{26}$ (72 months of follow-up) are the only two studies reported in humans. Clinical trials reported by Nakashima and Iohara $^{27}$ have initiated successful pulp regeneration with DPSCs. The ability to form bone, muscle, fibrous connective tissue, and nerves will be applied in the regenerative facial reconstruction for developmental defects of cleft palate and cleft lip in near future.

The regeneration of functional dental tissues form DSCs is more complicated than that of non-dental tissues. The research results from animal studies are not applicable for humans, because changes in dental structures are one of the cardinal signs of evolution and the maturation of dental tissues in animals is faster than that in humans. Animals and humans have anatomically dissimilar tooth making animal models limited in comparison with the human dental tissue. The time of initiation, eruption, and root completion of each tooth vary even in the human dentition with little correlation between them. ${ }^{24}$ Table 2 explains permanent tooth development from the time of initiation of tooth bud to root completion. Initiation indicates the availability of the active $\mathrm{DL}$ and the surrounding ectomesenchyme at different stages of life. The end of root completion conveys the end of availability of HERS, which are then replaced by ERM in humans. ${ }^{5}$ As deciduous teeth initiation and completion occurs during the fetal and in infant stage, the discussion of HERS and ERM as the cell source is under ethical issue and not with better scope. However, the deciduous teeth are loaded with DPSC, SHED, PDLSC, GMSC, ERM, and the SCAP till root completion. Exfoliating teeth (SHED) are an excellent source of DSC if the vital cells are preserved properly. The time lapse between the initiation and the completion of root indicates the time taken for normal tooth development in humans. The time taken for the permanent teeth completion varies from 7.8 to 12.5 years, which raises many questions regarding functional bioengineered tooth in human.

\section{OEpSCs AND THEIR POSSIBLE SOURCE}

The oral ectoderm ingrowth (DL) during development is the main source for the OEpSCs. The possible sources of OEpSCs in postnatal life include the active DL present in the retromolar region of the human jaw for 5-6 years, ${ }^{24}$ the remnants of DL in the gubernaculum cord (GC) present above any erupting tooth, ${ }^{24}$ the epithelial cell rests of Malassez $(\mathrm{ERM})^{3,24,28}$ covering the root of all teeth, and the reduced enamel epithelium (REE), ${ }^{24,29}$ which is a tissue layer covering the newly erupting tooth, the junctional epithelium $(\mathrm{JE})^{29}$ surrounding the neck of the teeth, and the HERS near the incomplete root ends.

Figure 2 shows the location of DSCs of mesenchymal and epithelial origin (OEpSCs) in postnatal life. This schematic presentation is the histology to be expected in a 12-13-yearold human in the posterior mandible molar region indicating the different stages of odontogenesis for different teeth. The availability of OEpSCs at this stage of life is expected from remnants of DL, GC, ERM, SCAP, and JE where JE is derived from REE. OEpSCs can also possibly become trapped in the dental pulp region and may initiate the formation of dentin inside pulp commonly known as pulp stones.

\section{Dental Lamina (DL) and Gubernacular Cord (GC)}

The DL is a continuous thickening of the oral ectoderm, forming an ingrowth into the mesenchyme in the region of the future dental arch at 45-48 days of human intrauterine life to develop tooth buds. ${ }^{24} \mathrm{DL}$ acquires this potential for odontogenesis at 30-35 days of development but collaborates with the underlying ectomesenchyme by 52-56 days of development. ${ }^{24,29}$ The ectomesenchyme gains the power to induce odontogenesis from dental and non-dental epithelium (and even from outer skin). ${ }^{24,29}$ Overexpression of DL is correlated with supernumerary tooth formation ${ }^{30}$ and also with abnormal manifestations of odontogenic cysts and tumor formation. ${ }^{31}$ Animal studies have proven the availability of the active DL, even in the edentulous space (diastema) of the jaws and its ability to form a bud without progressing. It has been shown that knockout mutations of genes required for odontogenesis cannot prevent the formation of dental epithelium. ${ }^{32,33}$ Additionally, it has been suggested that evolutionarily lost teeth can reappear, running counter to Dollo's law of the irreversibility of evolution..$^{34,35}$ This proves the persistence and potential of the odontogenic epithelium for millions of years.

The human DL yields permanent tooth buds in different stages of life, indirectly indicating that the DL becomes active in different stages of life (listed in Table 2). After tooth bud formation, the DL is programmed for apoptosis. There 
Table 2 Permanent dentition: the timing of tooth initiation and root completion and the time elapsed between stages ${ }^{\mathrm{a}, \mathrm{b}}$

\begin{tabular}{|c|c|c|c|c|c|c|c|}
\hline \multirow[t]{2}{*}{ Permanent teeth } & \multirow[t]{2}{*}{ Initiation } & \multicolumn{2}{|c|}{$\begin{array}{l}\text { Emergence into the } \\
\text { oral cavity (years) }\end{array}$} & \multicolumn{2}{|c|}{$\begin{array}{l}\text { Root completion } \\
\text { (years) }\end{array}$} & \multicolumn{2}{|c|}{$\begin{array}{l}\text { Time elapsed from } \\
\text { initiation to emergence (years) }\end{array}$} \\
\hline & & Male & Female & Male & Female & Male & Female \\
\hline \multicolumn{8}{|l|}{ Incisor } \\
\hline \multicolumn{8}{|l|}{ Upper } \\
\hline C & 18-20th week of IUL & 8.3 & 7.4 & 10.6 & 9.3 & 8.9 & 8 \\
\hline L & & 9.1 & 8.1 & 11.1 & 9.7 & 9.9 & 8.7 \\
\hline \multicolumn{8}{|l|}{ Lower } \\
\hline C & & 7.3 & 6.7 & 9.2 & 8.1 & 7.9 & 8.3 \\
\hline L & & 8.1 & 7.3 & 9.9 & 8.8 & 8.7 & 7.9 \\
\hline \multicolumn{8}{|l|}{ Canine } \\
\hline Upper & & 11 & 9.4 & 13.7 & 11.9 & 11.6 & 10 \\
\hline Lower & & 10.9 & 9.2 & 13.5 & 11.4 & 11.5 & 9.8 \\
\hline \multicolumn{8}{|l|}{ Premolar } \\
\hline \multicolumn{8}{|l|}{ Upper } \\
\hline 1 & & 11.1 & 9.7 & 13.5 & 11.8 & 11.7 & 10.2 \\
\hline 2 & & 11.6 & 10.6 & 13.8 & 12.6 & 12 & 11 \\
\hline \multicolumn{8}{|l|}{ Lower } \\
\hline 1 & & 11.2 & 9.9 & 13.3 & 11.9 & 11.8 & 10.5 \\
\hline 2 & & 11.9 & 10.6 & 14 & 12.8 & 12.5 & 11 \\
\hline \multicolumn{8}{|l|}{ Molar } \\
\hline \multicolumn{8}{|l|}{ Upper } \\
\hline 1 & 20th week of IUL & 7.8 & 7.2 & 10.1 & 9.2 & 8.2 & 7.8 \\
\hline 2 & First year of PNL & 12.4 & 11.8 & 14.6 & 13.6 & 12.4 & 11.8 \\
\hline 3 & $5-6$ years of life & 17.4 & 17.8 & 18.2 & 18.8 & 12 & 12.2 \\
\hline \multicolumn{8}{|l|}{ Lower } \\
\hline 1 & 20th week of IUL & 7.8 & 7.2 & 10 & 9.2 & 8.2 & 7.8 \\
\hline 2 & First year of PNL & 12.5 & 11.8 & 14.8 & 13.8 & 12.5 & 11.8 \\
\hline 3 & 5-6 years of life & 17.4 & 17.7 & 18.3 & 18.3 & 12 & 12.2 \\
\hline
\end{tabular}

${ }^{\mathrm{a} C}$, Central; L, Lateral; IUL, Intrauterine Life; PNL, Postnatal Life.

${ }^{\mathrm{b}}$ This table explains permanent tooth development from the time of initiation of tooth bud to root completion. Initiation indicates the availability of the active DL and the surrounding ectomesenchyme at different stages of life. The end of root completion conveys the end of availability of HERS and then replaced by ERM in human. ${ }^{5}$ As deciduous teeth initiation and completion occurs during the fetal and in infant stage, discussing them as a source is under ethical issue, and hence, it has not been considered in the table. The time lapse between the initiation and the completion of root indicates the time taken for normal tooth development in humans. The permanent teeth completion varies from 7.8 to 12.5 years. This table is prepared from the available sources related to the study on the stages of tooth development at different age. ${ }^{24}$ As the population-based differences have been very well noticed with tooth development, differences can be noticed in different populations in the age of eruption and completion. DL, dental lamina; ERM, epithelial cell rests of Malassez; HERS, Hertwig's epithelial root sheath.

may be certain remnants of the DL present in the dental arch, called 'cell rests of Serres' (Figure 3), to which many odontogenic tumors and cysts of the jaws are attributed.
These remnants detach from the stalk-like extension provided for the tooth bud by replacing the tissue with the fibrous condensation known as the GC, which aids tooth eruption. 


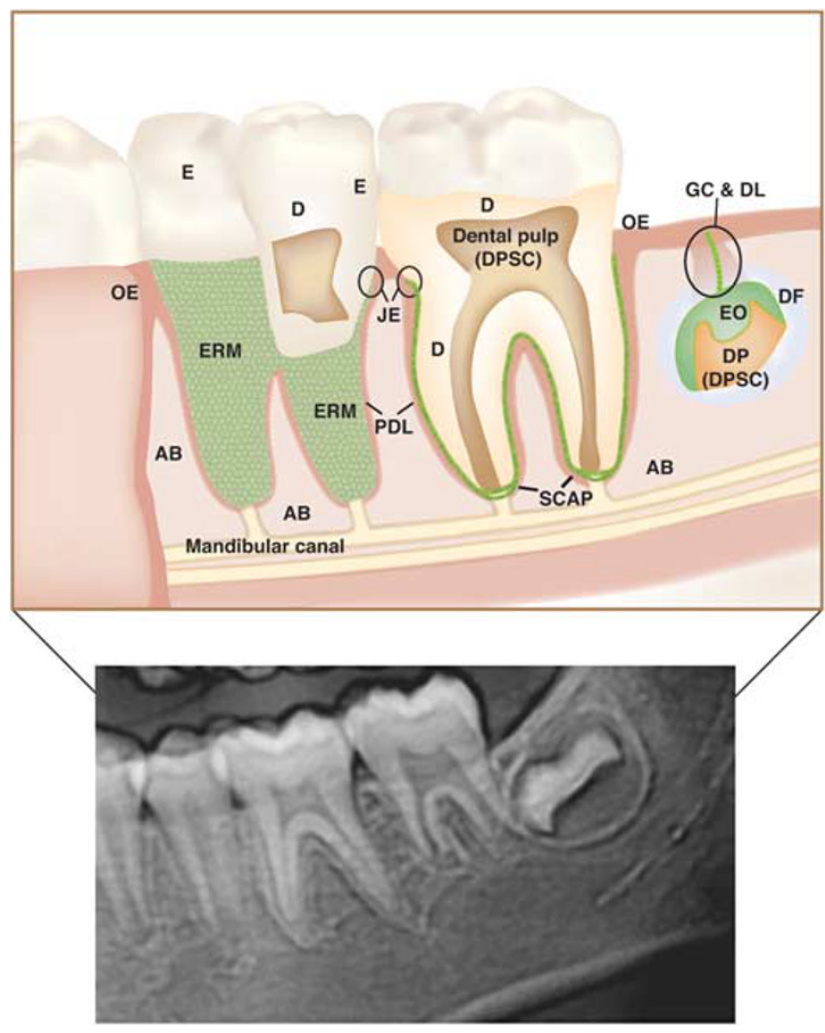

Figure 2 Radiograph and schematic representation of the posterior region of mandible of 12 and half-year-old child. Location of the dental stem cells of mesenchymal and epithelial origin in postnatal life is shown where the different levels of tooth development in the molar region of the posterior mandible reveal epithelial stem cells (OEpSCs) and mesenchymal stem cells in postnatal life. The webbed presentation expected from the ERM around the root, SCAP in the unclosed root apex, the EO of the developing tooth, DL remnants in the GC, and the JE derived from REE all represent odontogenic epithelial stem cells (OEpSCs). OEpSCs can also become trapped in the dental pulp region very minimally and may initiate the formation of pulp stones. $A B$, alveolar bone; C, cementum; D, dentin; DF, dental follicle; DL, dental lamina; DP, dental papilla; DPSCs, dental pulp stem cells; E, enamel; EO, enamel organ; ERM, epithelial rests of Malassez; GC, gubernacular cord; JE, junctional epithelium; OE, outer/oral epithelium; PDL, periodontal ligament; REE, reduced enamel epithelium; SCAP, stem cells of apical papilla.

This cord-like fibrous tissue is reported to contain remnants of the DL. ${ }^{24}$ The pericoronal tissues of the third molar region, which includes the GC, have been best explored, including the potential for odontogenic epithelial cell proliferation, associated histopathological changes in asymptomatic impacted molars. ${ }^{36}$ The retromolar area is the most common site for pathology reported in the jaw. ${ }^{37}$ It is suggested that the periodic clinical evaluation of asymptomatic impacted molars and their removal, including removal of the pericoronal tissue, is important to prevent complications. The presence of embryonic-stage DL in the retromolar area in the postnatal oral environment, which is exposed to unavoidable physical, mechanical, and chemical (microbial and inflammatory cytokines) trauma, can explain the extensive pathology

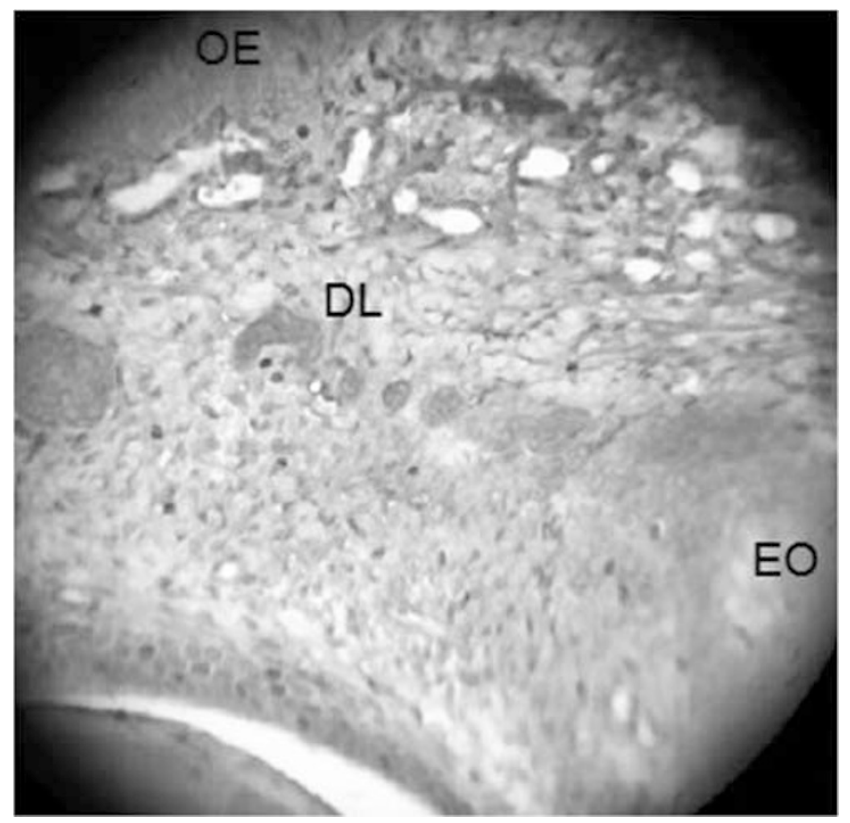

Figure 3 Remnants of DL or the 'cell rests of Serres.' The DL is the band of ingrowth of the outer $\mathrm{OE}$ inside the mesenchyme for the tooth bud formation. The continuity of the $\mathrm{OE}$ and the $\mathrm{DL}$ is obvious during the early stage of the development. The continuity will be disturbed because of the initiated apoptosis at later stage. The ultimate goal of the $\mathrm{DL}$ is tooth bud formation in the respective region of the different teeth at different stage of life. The DL cells are to be eliminated by apoptosis after the tooth bud formation in respective regions. However, a few of them can be trapped at the subepithelial or intra-alveolar level and may be responsible for many odontogenic epithelial tumors and cysts. These remnants of DL are named 'cell rests of Serres.' The stem cell population in these rests are odontogenic epithelial stem cells (OEpSCs). DL, dental lamina; EO, enamel organ; $\mathrm{OE}$, oral epithelium.

reported. These DL rests may represent a new source of OEpSCs.

There are many wisdom teeth removed owing to a lack of space and associated complications (1.3 million/year in the USA). ${ }^{38}$ Third molar tooth germ isolated from human donors has been verified for potential use as another source of embryonic stem cell-like cells, with fewer transgenic manipulations required for induction. ${ }^{39}$ Studies have even verified that dissociated tooth germ maintains its odontogenic potential upon re-aggregation. ${ }^{40}$ Research on dental tissue during organogenesis will settle debates about the development of many organs undergoing the same interactions if performed systematically at the interdisciplinary level. Currently, no research has testified the viability of the DL in postnatal life.

The isolation of OEpSCs from the active DL (from young children) raises ethical issues. Tracing the 'cell rests of Serres' is difficult because their persistence is questionable, but the GC can be easily located on the gingiva over the erupting tooth (Figure 2). The time of the tooth eruption reveals the availability of the GC (Table 2). The quantity and quality of OEpSCs from the GC must be investigated, because not much 
relevant research has been performed yet regarding the availability of the stem cells. Further research will unveil the contents and importance of OEpSCs on GC in tooth eruption, which is crucial to be explored regarding unerupted or impacted tooth. ${ }^{41}$

The unique feature of the upper four incisors is that they originate from the frontal lobe along with the forebrain, sharing the embryonic cell source (the neural ectoderm cells and CNCCs) of ocular development. Melanotic ectodermal tumors of infancy or retinal anlage tumors occur in the upper jaw anterior region, which histologically mimics the neural retina and the retinal-pigmented epithelium. ${ }^{42}$ This may indicate tumor development that recapitulates the retina from an earlier developmental stage. Whether the primitive ocular tissue becomes trapped and travels with the dental arch or the dental epithelium dedifferentiates to acquire a primitive nature has not been investigated systematically. If the GC and ERM from these areas are proved to contain OEpSCs, this might lead to successful formation of the pigmented and neural elements of retinal tissue. Mead et $a l^{43}$ reported that the DPSCs (mesenchymal cells) promote the regeneration of retinal ganglia. It is renowned that the head and neck regions share the common CNCC-derived ectomesenchyme during development.

\section{Epithelial Cell Rests of Malassez (ERM)}

After enamel formation, the outer and inner enamel epithelia of the EO at the cervical region join to form Hertwig's epithelial root sheath (HERS) to assist in root formation, and they will later cease to exist owing to apoptosis. ${ }^{5,24} \mathrm{~A}$ few cells are trapped in the periodontal ligament space close to the root surface, serving as the ERM. ${ }^{5,44}$ These cell rests are associated with many cysts and tumors of odontogenic epithelial origin. ${ }^{45}$ Recent research has demonstrated the role of the ERM in contributing to periodontal regeneration and the turnover of ERM cells throughout life. ${ }^{46}$ The ERM exhibit a unique ability called the EMT. These cells are epithelial cells but live in the connective tissue environment with the ability to differentiate into mesenchyme-related tissues by losing their basal lamina. ${ }^{17,46}$ These abilities raise the question of whether the cells should be named as cell rests.

The ability of the ERM to undergo EMT, their multipotentiality, and their differentiation into ameloblast-like cells have been studied by Xiong et al. ${ }^{17}$ An ERM cell line from human periodontium has been successfully established for further research. ${ }^{47}$ The millions of extracted teeth can be collected for the ERM to study the EMT, which is a vital process happening during development, tissue repair, and cancer generation.

If the teeth extraction is performed before root completion for orthodontic purposes or because of pathologically impacted teeth, active HERS can be collected from the root tip. ERM can be collected from the root surface of any extracted teeth or from the socket lining. ${ }^{47}$

\section{Reduced Enamel Epithelium (REE) and Junctional Epithelium (JE)}

After the completion of enamel formation, the EO collapses into a REE, which covers the enamel and prevents the dental follicular cells from forming a hard tissue over it and facilitates the eruption of the tooth by producing proteolytic enzymes to clear the surrounding tissues. ${ }^{24}$ REE becomes part of the JE once the tooth erupts inside the oral cavity. ${ }^{48} \mathrm{REE}$ is the lining epithelium for dentigerous cysts, the most common type of odontogenic cyst in young adults. ${ }^{37}$ The JE of bioengineered tooth was proven to be odontogenic in origin and to have epithelial stem cell potential reported by Yajima-Himuro et al ${ }^{49}$ in 2014.

REE can be obtained from the tissue covering any erupting crown and from the JE of erupting or recently erupted teeth. ${ }^{24}$ For use as a source of REE, the JE should be undisturbed by any disease process or surgical intervention; otherwise, the sulcular epithelium might have replaced the REE. The tooth development stages at 4-6 years in the human anterior mandible demonstrate their relationships between the developing permanent tooth and the resorbing deciduous teeth. ${ }^{24}$ OEpSCs are present in the REE, HERS, and in the DL remnants in the GC. DPSCs are present in the DP and DF of the developing permanent tooth and in the pulp of the resorbing deciduous teeth. The close proximity of the resorbing roots with open pulp and the REE can explain the possible amalgamation of OEpSCs with the pulp of the deciduous teeth during exfoliation.

\section{VIABILITY OF THE FUTURE PROSPECTS OF OEpSCs}

Studies regarding the potential of OEpSCs to be present in postnatal DL, REE, and JE have been discussed by several investigators. ${ }^{24-47}$ The GC could be one more source that is yet to be explored.

Studies have also focused on the odontogenic potential of non-dental structures from the oral cavity and from extra-oral structures. The studies reporting the odontogenic potential of the oral mucosa ${ }^{50}$ raise the question whether the remnants of DL ('cell rests of Serres') may be trapped in the oral mucosa. A report about stem cells from human exfoliated deciduous teeth (SHED-mesenchymal) containing epithelial stem cells ${ }^{51}$ again raises the possibility of entrapment of REE of the successor teeth below the deciduous teeth and also entrapment of epithelial cells inside the pulp during odontogenesis, which is a common finding correlated with the pathogenesis of pulp stone formation. ${ }^{52}$ This also is another possible source of OEpSCs. Wang et $a l^{53}$ reported that the inducted human keratinocytes form enamel by turning into ameloblasts in 2010.

Studies addressing epithelial stem cells in the formation of ameloblast or ameloblast-like cells and their ability have examined in bone marrow stem cell-derived ameloblast-like cells, ${ }^{54}$ enamel formation by sub-cultured odontogenic cells from the porcine $\mathrm{EO},{ }^{55}$ fully functional tooth regeneration from the mouse tooth germ, ${ }^{56}$ human adipose-derived stem cells induced to form a tooth bud, ${ }^{57}$ human gingival tissue as 
a source for whole tooth bioengineering, ${ }^{58}$ the self-renewal and multilineage potential of dental epithelial cells, ${ }^{59}$ the formation of epithelium by induced pluripotent stem cells from dental mesenchymal cells, ${ }^{60}$ non-keratinocyte cells induced to form dental epithelial cells, ${ }^{53}$ and characterization of dental epithelial cells, ${ }^{61}$ in addition to many detailed reviews of tooth bioengineering. ${ }^{61,62}$ Existing reports of in vitro and in vivo studies in animal and human tissues have provided the information necessary for further research to reach the bioengineered tooth popularly referred as the third dentition. OEpSC-derived enamel for clinical trials will be studied in the near future.

\section{OEpSCs in Tissue Engineering and Regenerative Medicine}

The ultimate goal of the use of OEpSCs in tissue engineering and regenerative medicine is the formation of enamel identical to natural enamel for the bio-tooth, which will become a reality in the near future.

Ikeda $e t a l^{56}$ reconstructed the fully functional bioengineered tooth by transplanting the bioengineered tooth germ into the jaw bone of mouse. The tooth germ was reconstructed with the original tooth germ derived from epithelial and MSCs. They suggested the use the third molar tooth germ for tooth engineering in human. Sonoyama et a ${ }^{63}$ also engineered fully functional tooth with the biomaterial scaffold to develop the dentin and root below the artificial porcelain crown using the SCAP cells in swine. Functional bio-tooth engineering for human use has been challenged by nature because of the limited sources of OEpSCs. The difficulty faces in (i) achieving the desired size and shape of the enamel, dentin and root/roots with the perfect junctions and periodontal attachments, (ii) the long duration of tooth formation (Table 2), and (iii) establishing the functional occlusion. Finally, the most challenging aspect will be performing clinical trials in the human oral environment augmented with functional and microbial insults.

The enamel is a nonliving tissue similar to hair and nails, supported by the underlying connective tissue. Dentin is the supportive tissue; serving with a junction having merely physical and mechanical in nature. ${ }^{24}$ There are 5-12 million enamel rods in the permanent teeth and 50000-90 000s of dentinal tubules per square millimeter of dentin. ${ }^{64}$ This highly organized structure of teeth must be formed by the synchronized reciprocal work differentiating at different times and rates. Re-creation of the enamel is possible only with transplantation of naturally formed tooth germ that has already been completely programmed. Third dentition remains the dream of dentists.

One advantage of the nonliving nature of the enamel is that a scaffold of the desired shape can be designed with strong biocompatible nanoparticles and then incorporated with the bioengineered enamel during tooth engineering. Because the desired shapes can be produced, exciting possibilities in regenerative therapy for dental cavities include inlays of bioengineered enamel (such as computer-aided design and computer-aided manufacturing porcelain inlays).

OEpSCs will have a major role in the tooth engineering where the crucial role of OEpSCs during the formation of dentin, root, cementum, and periodontal tissues can be used to regenerate these tissues. Research can be designed for cementum-coated implants to simulate the periodontium, pulp-capping biomaterials enriched with OEpSCs for dentin formation, and guided tissue regeneration of the periodontium with OEpSC reinforcement.

\section{Non-dental Derivation from OEpSCs}

Non-dental derivations from oral mucosal epithelial stem cells are routinely successful. Clinical trials have been reported for the use of tissue-engineered oral mucosal epithelium to fabricate corneal epithelium in humans by Nishida et al, ${ }^{65}$ and successful animal trials have been reported to produce esophageal epithelium by Takagi et $a l^{66}$ and tracheal epithelium by Kanzaki et al.$^{67}$ However, bioengineered teeth and enamel have not yet been generated for humans.

Endodermal-epithelial interactions in the posterior molars have been discussed for many years in the context of odontogenesis. If these interactions are proved, the possibilities of endodermal organ engineering from DSCs will be undeniable. ${ }^{68}$ The regeneration of non-dental tissues of critical value, such as the cornea, cardiac cells, neurons, pancreatic islets, and retina, from oral and DSCs will have favorable results in the near future because of their embryonic origin. ${ }^{18-23,43}$

The current treatment protocols for dental defects by either the fillings with biocompatible materials or the tooth replacement with dental implants are well established with standard techniques, which are economically affordable and ethically safe. Clinical studies on human dental tissue regeneration should target acceptable and easy procedures for application in regenerative medicine with nano-structured materials as reported by Mitsiadis et al. ${ }^{69}$ and Mitsiadis and Papagerakis. ${ }^{70}$

\section{CONCLUSIONS AND PERSPECTIVES}

DSCs will provide promising results in regenerative therapy, not just for dental structures but also for many vital structures. OEpSCs sourcing from DL, ERM, REE, and JE have yielded positive results, but the most promising OEpSC source is the ERM. The GC has yet to be explored. The GC from the upper anterior region must be investigated for the potential to form retinal tissue. The DL is the only embryonic tissue available late in postnatal life in the third molar region that can be used for studies related to organogenesis. Exfoliating teeth in children and the third molar or its tooth germ with the pericoronal tissue of adults are the best sources of OEpSCs. Public awareness can be generated to encourage their preservation in tooth banks. Promising research areas for study in the near future are the nanomaterials incorporated with OEpSCs for regenerative therapy in dentistry and the ability of the ERM to form retina and to undergo the EMT 
owing to their developmental biological properties. A bioengineered tooth is possible only by using the fully programmed tooth germ. Human clinical trials with wellcontrolled and monitored research works with transplanted tooth germ may provide remarkable results. Endodermderived oral tissues with stem cell potential in humans may help with the tissue engineering of endodermal cells such as pancreatic islets. The tissue available in dental structures is likely sufficient to form many organs, not just bioengineered teeth.

Stem cells have yielded promising therapeutic results for many incurable diseases. Soon, thanks to stem cell therapy, dental filling materials will undergo revolutionary changes. Concurring expert scientists from different fields joining together and conducting concurrent studies with the same protocols in many parts of the world will lead us to rapid success. The suggestions of this article about the search for sources of OEpSCs may project like hypothesis but research on them will also hopefully lead us to new horizons.

\section{ACKNOWLEDGMENTS}

This research was partially supported by the Ministry of Science and Technology, Taiwan, under the grant numbers 103-2120-M-008-001 and 102-2221-E-008-112-MY2. This work was also supported by the Landseed Hospital project (NCU-LSH-102-A-003 and 103LSH-NCU-1), the National Defense Medical Center Project (102NCU-NDMC-01), and the Cathay General Hospital Project (102NCU-CGH-02, 103CGH-NCU-A3, CGH-MR-A10204 and CGH-MR-A10301). A Grant-in-Aid for Scientific Research (number 15K06591) from the Ministry of Education, Culture, Sports, Science, and Technology of Japan is also acknowledged. We acknowledge the International High Cited Research Group (IHCRG \#14-104), Deanship of Scientific Research, King Saud University, Riyadh, Kingdom of Saudi Arabia.

\section{DISCLOSURE/CONFLICT OF INTEREST}

The authors declare no conflict of interest.

1. Daley GQ, Scadden DT. Prospects for stem cell-based therapy. Cell 2008;132:544-548.

2. Nadig RR. Stem cell therapy - Hype or hope? A review. J Conservative Dent 2009;12:131-138.

3. Bluteau G, Luder HU, De Bari C et al. Stem cells for tooth engineering. Eur Cells Mater 2008;16:1-9.

4. Jussila M, Thesleff I. Signaling networks regulating tooth organogenesis and regeneration, and the specification of dental mesenchymal and epithelial cell lineages. Cold Spring Harb Perspect Biol 2012;4:a008425.

5. Thomas HF. Root formation. Int J Dev Biol 1995;39:231-237.

6. Huang $A H, C h e n ~ Y K$, Lin LM et al. Isolation and characterization of dental pulp stem cells from a supernumerary tooth. J Oral pathol Med 2008:37:571-574.

7. Arora V, Arora P, Munshi AK. Banking stem cells from human exfoliated deciduous teeth (SHED): saving for the future. J. Clin. Pediatr Dent. 2009;33:289-294.

8. Alge DL, Zhou D, Adams LL et al. Donor-matched comparison of dental pulp stem cells and bone marrow-derived mesenchymal stem cells in a rat model. J Tissue Eng Reg Medicine 2010;4:73-81.

9. Gronthos S, Mankani M, Brahim J et al. Postnatal human dental pulp stem cells (DPSCs) in vitro and in vivo. Proc Natl Acad Sci USA 2000;97: 13625-13630.

10. Gronthos S, Brahim J, Li W et al. Stem cell properties of human dental pulp stem cells. J Dent Res 2002;81:531-535.

11. Graziano A, d'Aquino R, Cusella-De Angelis MG et al. Scaffold's surface geometry significantly affects human stem cell bone tissue engineering. J Cell Physiol 2008;214:166-172.
12. Miura $M$, Gronthos $S$, Zhao $M$ et al. SHED: stem cells from human exfoliated deciduous teeth. Proc Natl Acad Sci USA 2003;100: 5807-5812.

13. Handa K, Saito M, Tsunoda A et al. Progenitor cells from dental follicle are able to form cementum matrix in vivo. Connect Tissue Res 2002;43: 406-408.

14. Hasegawa $\mathrm{M}$, Yamato $\mathrm{M}$, Kikuchi $\mathrm{A}$ et al. Human periodontal ligament cell sheets can regenerate periodontal ligament tissue in an athymic rat model. Tissue Eng 2005;11:469-478.

15. Huang GT, Sonoyama, Liu WY et al. The hidden treasure in apical papilla: the potential role in pulp/dentin regeneration and bioroot engineering. J Endodont 2008;34:645-651.

16. Zhang $\mathrm{Q}$, Shi $\mathrm{S}$, Liu $\mathrm{Y}$ et al. Mesenchymal stem cells derived from human gingiva are capable of immunomodulatory functions and ameliorate inflammation-related tissue destruction in experimental colitis. J Immunol 2009;183:7787-7798.

17. Xiong J, Mrozik K, Gronthos S et al. Epithelial cell rests of Malassez contain unique stem cell populations capable of undergoing epithelial-mesenchymal transition. Stem Cells Dev 2012;21: 2012-2025.

18. La Noce M, Paino F, Spina A et al. Dental pulp stem cells: state of the art and suggestions for a true translation of research into therapy. J Dent 2014;42:761-768.

19. Kim RH, Mehrazarin S, Kang MK. Therapeutic potential of mesenchymal stem cells for oral and systemic diseases. Dent Clin North Am 2012;56: 651-675.

20. Bakopoulou A, Leyhausen G, Volk J et al. Comparative analysis of in vitro osteo/odontogenic differentiation potential of human dental pulp stem cells (DPSCs) and stem cells from the apical papilla (SCAP). Arch Oral Biol 2011;56:709-721.

21. Sunil $\mathrm{P}$, Manikandhan $\mathrm{R}$, Muthu $\mathrm{M}$ et al. Stem cell therapy in oral and maxillofacial region: An overview. J Oral Maxillofac Pathol 2012;16: 58-63.

22. Rai S, Kaur M, Kaur S. Applications of stem cells in interdisciplinary dentistry and beyond: an overview. Ann Med Health Sci Res 2013;3: 245-254.

23. Egusa $\mathrm{H}$, Sonoyama $\mathrm{W}$, Nishimura $\mathrm{M}$ et al. Stem cells in dentistry-Part II: Clinical applications. J Prosthodont Res 2012;56:229-248.

24. Nanci A. Ten Cate's Oral Histology: Development Structure and Function, 8th edn. Elsevier: St Louis, MO, 2013.

25. d'Aquino R, De Rosa A, Lanza $V$ et al. Human mandible bone defect repair by the grafting of dental pulp stem/progenitor cells and collagen sponge biocomplexes. Eur Cells Mater 2009;18:75-83.

26. Feng $\mathrm{F}$, Akiyama $\mathrm{K}$, Liu $\mathrm{Y}$ et al. Utility of PDL progenitors for in vivo tissue regeneration: a report of 3 cases. Oral Dis 2010;16:20-28.

27. Nakashima M, lohara K. Mobilized dental pulp stem cells for pulp regeneration: initiation of clinical trial. J Endodont 2014;40:S26-S32.

28. Standring S. Gray's Anatomy, Anatomical Basis of Clinical Practice. London: Elsevier Churchill Livingstone: London. 2005;39.

29. Rajkumar K, Ramya R. Text Book of Oral Anatomy, Histology, Physiology \& Tooth Morphology. Gurgaon, India: Wolters Kluwer Health. 2012.

30. Koussoulakou DS, Margaritis LH, Koussoulakos SL. A curriculum vitae of teeth: evolution, generation, regeneration. Int J Biol Sci 2009;5: 226-243.

31. Jheon AH, Seidel K, Biehs B et al. From molecules to mastication: the development and evolution of teeth. Wiley Interdiscip Rev Dev Biol 2013;2:165-182.

32. Jackman WR, Stock DW. Transgenic analysis of Dlx regulation in fish tooth development reveals evolutionary retention of enhancer function despite organ loss. Proc Natl Acad Sci USA 2006;103: 19390-19395.

33. Mitsiadis TA, Cheraud Y, Sharpe P et al. Development of teeth in chick embryos after mouse neural crest transplantations. Proc Natl Acad Sci USA 2003;100:6541-6545.

34. Rao PV, Chidzonga M. Supernumerary teeth: Literature review. Cent Afr J Med 2001;47:22-26.

35. Dollo L. Les lois de l'évolution. Bull Soc Belge Geol Pal Hydr 1893;7: 164-166.

36. Adeyemo WL. Do pathologies associated with impacted lower third molars justify prophylactic removal? A critical review of the literature. Oral Surg Oral Med Oral Pathol Oral Radiol Endod 2006;102:448-452. 
37. Neville BW, Damm DD, Allen CM. Odontogenic Cysts and Tumors. Philadelphia: Saunders: Philadelphia. 2009;3:pp678-731.

38. American Dental Association 2005-06 Survey of Dental Services Rendered. Chicago: American Dental Association: Chicago. 2007.

39. Yalvac ME, Ramazanoglu $M$, Rizvanov $A A$ et al. Isolation and characterization of stem cells derived from human third molar tooth germs of young adults: implications in neo-vascularization, osteo-, adipo- and neurogenesis. Pharmacogenomics J 2010;10:105-113.

40. Hu X, Lin C, Wang B et al. Dissociated mouse tooth germ epithelial cells retain the expression of tooth developmental genes during reaggregation process. Chinese J Biotech 2010;26:1690-1695.

41. Ferreira DCA, Fumes AC, Consolaro A et al. Gubernacular cord and canal - does these anatomical structures play a role in dental eruption? RSBO 2013:10:167-171.

42. Lamping KA, Albert DM, Lack E et al. Melanotic neuroectodermal tumor of infancy (retinal anlage tumor). Ophthalmology 1985;92: 143-149.

43. Mead B, Logan A, Berry $M$ et al. Intravitreally transplanted dental pulp stem cells promote neuroprotection and axon regeneration of retinal ganglion cells after optic nerve injury. Invest Ophthalmol Vis Sci 2013;54:7544-7556

44. Keinan D, Cohen RE. The significance of epithelial rests of Malassez in the periodontal ligament. J Endod 2013;39:582-587.

45. Lin LM, Huang GT, Rosenberg PA. Proliferation of epithelial cell rests, formation of apical cysts, and regression of apical cysts after periapical wound healing. J Endod 2007;33:908-916.

46. Rincon JC, Young WG, Bartold PM. The epithelial cell rests of Malasseza role in periodontal regeneration? J Periodontal Res 2006;41:245-252.

47. Nam H, Kim JH, Kim JW et al. Establishment of Hertwig's epithelial roo sheath/epithelial rests of Malassez cell line from human periodontium. Mol Cells 2014:37:562-567.

48. Gao Z, Mackenzie IC. Patterns of phenotypic expression of human junctional, gingival and reduced enamel epithelia in vivo and in vitro. Epithelial Cell Biol 1992;1:156-167.

49. Yajima-Himuro S, Oshima M, Yamamoto G et al. The junctional epithelium originates from the odontogenic epithelium of an erupted tooth. Sci Rep 2014;4:4867.

50. Nakagawa $\mathrm{E}$, Itoh $\mathrm{T}$, Yoshie $\mathrm{H}$ et al. Odontogenic potential of post-natal oral mucosal epithelium. J Dent Res 2009;88:219-223.

51. Nam H, Lee G. Identification of novel epithelial stem cell-like cells in human deciduous dental pulp. Biochem Biophys Res Commun 2009:386:135-139.

52. Goga R, Chandler NP, Oginni AO. Pulp stones: a review. Int Endod J 2008:41:457-468.

53. Wang B, Li L, Du S et al. Induction of human keratinocytes into enamelsecreting ameloblasts. Dev Biol 2010;344:795-799.
54. Hu B, Unda F, Bopp-Kuchler $\mathrm{S}$ et al. Bone marrow cells can give rise to ameloblast-like cells. J Dent Res 2006;85:416-421.

55. Honda MJ, Shinohara Y, Hata Kl et al. Subcultured odontogenic epithelial cells in combination with dental mesenchymal cells produce enamel-dentin-like complex structures. Cell Transplant 2007;16: 833-847.

56. Ikeda $\mathrm{E}$, Morita $\mathrm{R}$, Nakao $\mathrm{K}$ et al. Fully functional bioengineered tooth replacement as an organ replacement therapy. Proc Natl Acad Sci USA 2009;106:13475-13480.

57. Ferro F, Spelat R, Falini $G$ et al. Adipose tissue-derived stem cell in vitro differentiation in a three-dimensional dental bud structure. Am J Pathol 2011;178:2299-2310.

58. Angelova Volponi A, Kawasaki M, Sharpe PT. Adult human gingival epithelial cells as a source for whole-tooth bioengineering. J Dent Res 2013;92:329-334.

59. Chang JY, Wang C, Jin C et al. Self-renewal and multilineage differentiation of mouse dental epithelial stem cells. Stem Cell Res 2013:11:990-1002.

60. Arakaki M, Ishikawa M, Nakamura $T$ et al. Role of epithelial-stem cell interactions during dental cell differentiation. J Biol Chem 2012;287: 10590-10601.

61. Chavez MG, Yu W, Biehs B et al. Characterization of dental epithelial stem cells from the mouse incisor with two-dimensional and threedimensional platforms. Tissue Eng Part C Methods 2013;19:15-24.

62. Mao JJ, Prockop DJ. Stem cells in the face: tooth regeneration and beyond. Cell Stem Cell 2012;11:291-301.

63. Sonoyama W, Liu Y, Fang D et al. Mesenchymal stem cell-mediated functional tooth regeneration in swine. PLOS ONE 2006;1:e79.

64. Kumar GS. Orban's Oral Histology and Embryology. New Delhi: Elsevier: New Delhi. 2011;13.

65. Nishida $\mathrm{K}$, Yamato $\mathrm{M}$, Hayashida $\mathrm{Y}$ et al. Corneal reconstruction with tissue-engineered cell sheets composed of autologous oral mucosal epithelium. New Engl J Med 2004;351:1187-1196.

66. Takagi $R$, Yamato $M$, Kanai $N$ et al. Cell sheet technology for regeneration of esophageal mucosa. World J Gastroenterol 2012;18: 5145-5150.

67. Kanzaki $M$, Yamato $M$, Kanai $N$ et al. Tissue engineered epithelial cell sheets for the creation of a bioartificial trachea. Tissue Eng 2006;12: 1275-1283.

68. Soukup V, Epperlein $\mathrm{HH}$, Horacek I et al. Dual epithelial origin of vertebrate oral teeth. Nature 2008;455:795-798.

69. Mitsiadis TA, Woloszyk A, Jimenez-Rojo L. Nanodentistry: combining nanostructured materials and stem cells for dental tissue regeneration. Nanomedicine-UK 2012;7:1743-1753.

70. Mitsiadis TA, Papagerakis P. Regenerated teeth: the future of tooth replacement? Regen Med 2011;6:135-139. 